\title{
Allocution d'ouverture
}

Anne-Marie Idrac

\section{OpenEdition}

Journals

Édition électronique

URL : https://journals.openedition.org/rhcf/672

DOI : 10.4000/rhcf.672

Éditeur

Rails \& histoire

Édition imprimée

Date de publication : 1 juin 2008

Pagination : 15-22

ISSN : 0996-9403

Référence électronique

Anne-Marie Idrac, " Allocution d'ouverture », Revue d'histoire des chemins de fer [En ligne], 39 | 2008, mis en ligne le 01 juin 2011, consulté le 22 avril 2022. URL : http://journals.openedition.org/rhcf/672 ; DOI : https://doi.org/10.4000/rhcf.672

Tous droits réservés 
Anne-Marie IDRAC

\section{Allocution d'ouverture}

\section{$M$} traits, sinon une rétrospective, du moins une prospective.

C'est le $20^{\mathrm{C}}$ anniversaire de votre association, ce sera aussi, le $1^{\text {er }}$ janvier 2008 , le $70^{\mathrm{e}}$ anniversaire de la SNCF. Nous avons choisi à cette occasion d'inviter le public à une exposition, au Grand Palais, qui porte précisément sur l'art et l'architecture liés au chemin de fer, empruntant le même chemin que celui qui nous a réunis dans ce lieu aujourd'hui.

2007, c'est aussi le 50 anniversaire du service Trans Europe Express entre Lyon et Milan, me dit-on, le $40^{\mathrm{e}}$ du Capitole à $200 \mathrm{~km} / \mathrm{h}$, le $20^{\mathrm{e}} \mathrm{du}$ TER, le $10^{\text {e }}$ de RFF...

Ce qui me semble intéressant dans cette histoire de notre entreprise, puisque jusqu'à l'an passé SNCF et chemin de fer en France étaient synonymes, c'est de comprendre la nature des liens entre mon entreprise, notre entreprise, et la société française, des liens qui sont tout à fait particuliers, très originaux, faits d'affection, quelquefois d'amour, mais, dans ce domaine, c'est un peu «je t’aime, moi non plus ». 
Des sept premières décennies de l'histoire de la SNCF, même si votre colloque n'en retient que deux, je souhaite retenir quatre éléments marquants.

Le premier, c'est que la SNCF est et a su rester populaire : c'est son premier lien avec la société française. Je crois que ceci est dû, en particulier, au modèle de développement qui a été choisi en France pour la grande vitesse, caractérisé par un très fort taux de remplissage qui implique des tarifs qui ne sont pas très élevés. Ce modèle est donc très différent de celui qu'ont adopté les autres pays européens. Ce modèle populaire concilie une rentabilité économique très forte avec le caractère démocratique souhaité pour le chemin de fer dans notre pays, a fortiori bien sûr pour l'entreprise publique que nous sommes. Ce choix de la politique de volume, qu'on a vu pour la grande vitesse, je souhaite le développer dans une large mesure, et valoriser dans l'imaginaire de l'entreprise, davantage qu'on ne l'a fait jusqu'ici, les activités de proximité, Transilien et les TER que je viens de mentionner, précisément en raison de ce côté populaire qui me paraît être très important dans notre identité passée et pour la préservation de notre identité à venir, puisque nous aurons à la défendre dans la concurrence.

Le deuxième élément, c'est bien sûr la fierté d'être pour notre pays, à travers le monde, une vitrine technologique. C'est l'excellence technologique des records du monde de vitesse sur rail successifs, comme le dernier en date sur la ligne du TGV Est Européen auquel plusieurs d'entre nous avons eu la chance de participer. Nous partageons cette vitrine technologique avec le constructeur Alstom, un partage qui va sans doute évoluer à l'avenir. En effet, Bombardier vend par exemple à présent des trains en Chine ; faut-il que la SNCF s'abstienne d'y accompagner Bombardier pour la raison que, pour l'instant, nous achetons des trains à grande vitesse Alstom en France?

Quoi qu'il en soit, cette excellence technologique me semble devoir s'ouvrir à présent, et de plus en plus, sur les services. Par exemple, nous avons investi l'année dernière une vingtaine de millions d'euros dans la connexion Internet Wifi à bord. Autre exemple, l'un des projets dans lesquels nous investissons le plus en ce moment est la dématérialisation des titres de transport. Par ailleurs, la période de crise que traverse la SNCF en ce moment [18-23 novembre 2007] nous aura au moins permis de gagner des points dans le domaine de l'information des clients.

Troisième élément historique qui se prolonge dans le présent, c'est la notion de responsabilité sociale. Nous avons fait procéder à une notation extra financière de la SNCF et j’ai choisi Vigéo pour ce faire. J'en aurai les résultats à la fin de 2007. La SNCF a toujours été, bien évidemment, une entreprise responsable, en particulier dans sa relation avec les territoires ; aujourd'hui 
elle développe sa responsabilité sociale du point de vue de l'accès de ses services aux personnes handicapées, du point de vue de la diversité des recrutements qui est une forme, je crois, moderne et nouvelle de cette responsabilité. C'est pour cela, et j'y reviendrai, que la notion de développement durable me parât extrêmement porteuse puisqu'elle lie tous les enjeux de responsabilité : économique, sociale, sociétale et environnementale.

Enfin, le dernier point de cette histoire, s'il n'a pas tout à fait la même valeur que les autres, est important, surtout à la gare d'Orsay : c'est le lien entre le train et la société via l'architecture et le patrimoine. Notre patrimoine architectural n'est pas figé. On voit bien, par exemple, ce que Jean-Marie Duthilleul a réussi à faire de la gare de Strasbourg, quelque chose de tout à fait extraordinaire qui l'a fait passer du XIX ${ }^{\mathrm{e}}$ au $\mathrm{XXI}^{\mathrm{e}}$ siècle.

Voilà donc une entreprise qui a gardé des liens et créé des liens avec ce qui bougeait dans la société française, liens que finalement l'aventure du TGV symbolise le mieux. Nous avons transformé la carte de France, en la rendant non plus lisible en kilomètres mais en heures de trajet, et, du coup, nous avons adapté les tarifs. C'est avec Jacques Fournier, je m'en souviens, quand j'étais commissaire du gouvernement auprès de lui, que nous avons transformé la tarification kilométrique en tarification, pour partie, à la vitesse, par un arrêté de 1994 qui porte mon nom.

La SNCF a projeté les Français dans leur propre modernité, nous avons projeté le ferroviaire dans un univers de services. Aujourd'hui, nous sommes dans la dimension européenne de cette modernité, liée au territoire. Je pense que le TGV Est Européen donne une dimension plus complète au TGV par son articulation avec le TER. Encore mieux que précédemment, les horaires et les gares ont été aménagés pour faciliter les correspondances. À Strasbourg, on privilégie très largement l'intermodalité avec les autres modes de transport : le tramway, le tram-train. Cette relation au territoire s'est, à mon avis, enrichie et atteint sa plénitude à la fois régionale et européenne dans le cas du TGV Est Européen où l'on retrouve le côté populaire de la grande vitesse, avec un taux de remplissage de $80 \%$ sur les liaisons vers Paris. Du côté des trains intersecteurs le résultat est moins probant, à ce stade, mais il est normal qu'il y ait moins de voyageurs qui aient envie d'aller de Strasbourg à Bordeaux. Il faut créer le produit, il faut créer le marché. Comme en outre la technologie du TGV Est Européen est tournée vers le service - y compris par l'architecture - je le vois comme le point d'articulation entre notre histoire et ce qu'il préfigure de notre avenir. 
Le chemin de fer en France, dans vingt ans, sera fort différent de ce que vous connaissez puisque vous aurez dans la salle, bien sûr des cheminots de la SNCF, et peut-être le président de la SNCF, peut-être même encore une présidente, mais aussi d'autres entreprises ferroviaires. Ce sera la première - très grande - différence avec aujourd'hui : le chemin de fer ne sera plus synonyme de SNCF.

De même il faut à présent préciser, quand on parle de cheminots, « cheminots de la SNCF ", si on veut être conforme à la réalité. Il y a d'autres cheminots que ceux dont je suis la patronne depuis maintenant dix-huit mois. Cela va faire bouger les choses, beaucoup de choses. J'ai dit récemment aux dirigeants du groupe SNCF, devant Jacques Barrot que j'avais invité, que nous sommes dans trois révolutions.

La première et la plus importante est évidemment l'entrée dans la concurrence intramodale. Que le chemin de fer soit en concurrence avec la voiture, avec le camion, avec l'avion n'est évidemment pas une nouveauté. Mais son avenir, c'est d'être en concurrence avec lui-même avec l'espoir, bien entendu, que cela stimule le marché et développe le chemin de fer pour toutes les entreprises concernées, comme cela a été le cas dans les pays voisins, en particulier en Grande-Bretagne ou en Allemagne, où l'on constate effectivement la stimulation du marché par la concurrence et le gain de parts de marché, notamment en Grande-Bretagne, du fret ferroviaire par rapport au fret routier. Cette concurrence nous amène à nous positionner, nous, c'est-à-dire l'entreprise, mais aussi tout son environnement : les pouvoirs publics, les politiques, les intellectuels, etc., car tous sont plus ou moins pris de court par la nécessité de trouver leur place dans des univers totalement nouveaux, comme cela a été le cas pour les télécommunications ou pour l'électricité. Mais si ces secteurs s'y sont préparés depuis plusieurs décennies, nous, à vrai dire, ne l'avons guère fait, parce qu'on n'a pas osé dire les choses ni les regarder en face, par peur. Cette concurrence sera très différente selon les produits, elle ne sera pas la même pour le fret et pour les transports de proximité ou pour le transport à longue distance. La concurrence portera aussi sur le matériel. La maintenance sera-t-elle effectuée par l'entreprise de transport ou par ses fournisseurs ? Elle va jouer aussi dans le domaine des infrastructures. Quelle sera la part des travaux d'infrastructure faits par des entreprises privées de travaux ferroviaires par rapport à l'entreprise intégrée SNCF ? Des univers nouveaux vont s'ouvrir, bien sûr il va falloir tirer au mieux notre épingle du jeu. Revenons à votre colloque qui porte, au-delà de la SNCF, sur le transport ferroviaire. 
La deuxième révolution est à mon avis celle qu'en France nous appelons le Grenelle de l'environnement et qui, à l'échelle mondiale, à l'échelle européenne en tout cas, se nomme le développement durable. Alors vous vous dites tous que le chemin de fer, ayant, comme le prédisait Louis Armand, survécu au $\mathrm{XX}^{\mathrm{e}}$ siècle, c'est à présent le mode de transport du $\mathrm{XXI}^{\mathrm{e}}$ siècle. Je pense effectivement, quand on se place à l'échelle mondiale, que certaines des évolutions conduisent à donner un très grand espoir au chemin de fer. J'en vois deux en particulier : le monde est en train de se structurer autour des ports et des grandes métropoles.

Le chemin de fer va très bien avec les ports et les grandes métropoles parce qu'il s'accorde avec la massification, qu'il s'agisse des containers pour les ports ou des forts volumes de personnes pour les grandes métropoles. Je résume certes à l'extrême, mais je crois que ce schéma est juste; dans le cas de la France, où nous avons très peu de ports, ou en tout cas peu de ports dont l'hinterland soit important, où les investissements dans ce domaine ont été faibles et où le contexte administratif reste complexe (par exemple quant à la propriété des voies des ports), ce lien privilégié entre ports et chemin de fer passera par le transport de marchandises à longue distance, en particulier par la containerisation; je pense ainsi être en mesure de doubler le trafic par transport combiné dans les quatre à cinq années qui viennent. Du côté des voyageurs, ce sera le développement du transport de proximité et de la grande vitesse. Les critères retenus pour le développement des lignes nouvelles sont la possibilité de conquérir des parts de marché par rapport à l'avion, la désaturation du réseau et les capacités des gares à gérer les nouveaux flux qu'elle va générer.

Cette révolution que représente le développement durable est, vraiment, j'en ai la conviction, très porteuse d'avenir, pour toutes les entreprises ferroviaires. Les dispositions qui viennent d'être annoncées, en particulier la fameuse taxe sur les camions que les cheminots attendaient depuis longtemps, va améliorer la compétitivité du fer en général et des entreprises ferroviaires par rapport au camion ; même si cette disposition n'améliorera pas la compétitivité relative de la SNCF par rapport aux autres entreprises ferroviaires, elle lui donnera un nouvel élan.

Le chemin de fer est un mode écologique parce qu'il est - nous sommes - un mode collectif, parce que nous massifions les flux ; en France, nous sommes un mode écologique parce que nous fonctionnons, plus ou moins, grâce à l'énergie nucléaire. La diversification de l'alimentation électrique m’oblige à dépenser beaucoup d'argent pour acheter des certificats verts, parce que je ne veux pas que le changement d'approvisionnement électrique ait de répercussion écologique. Nous devons bien entendu être exemplaires dans nos 
économies d'énergie, dans notre diversification des carburants et dans notre mode de gestion des rejets, du papier, etc. Le développement durable est un processus, non un état ; ce qui est important, c'est d'être et de devenir toujours davantage un acteur de ce développement durable.

La troisième révolution est d'ordre social et managérial. On le voit bien, il existe un décalage entre la vie à l'intérieur de ma société, de mon entreprise, et la vie de la société française. Ce décalage est manifesté, sans doute, par les difficultés actuelles à comprendre l'évolution du régime de retraite qui est ressentie par les cheminots comme une rupture du contrat. Je le ressens aussi, comme je ressens la revendication du monde extérieur qui nous dit en retour: «Vous êtes en train de rompre le contrat entre la société globale et la Société nationale des chemins de fer français. » Cette évolution sociale et managériale a donc une très grande importance - d'autant que la grève actuelle est l'antiGrenelle de l'environnement puisqu'on met par elle beaucoup de $\mathrm{CO}^{2}$ dans l'atmosphère et de camions sur les routes.

Ce qui est en train de se passer de ce point de vue est dramatique : on en vient à un sujet historique qui n'est pas simplement le problème des dirigeants de la SNCF. Si le port de Marseille est malheureusement contourné par des autoroutes maritimes qui vont de Gênes à Barcelone, c'est pour des raisons sociales. Ce sujet dépasse donc le seul problème interne de l'entreprise. Si les chargeurs sont réticents à se rapprocher de la SNCF c'est parce que, pour une large part, ils ne nous font pas confiance en termes sociaux. Entendre des chargeurs dire : «Heureusement que j’ai adopté le camion dans mon organisation logistique depuis plusieurs années parce qu'au moins je peux approvisionner ainsi mes usines, mes clients ou être approvisionné par mes fournisseurs » est la traduction d'un phénomène d'envergure. La troisième révolution que je dois mener, qui relève de ma responsabilité puisque je me trouve là, moi, à ce moment-là, c'est donc de rapprocher l'économique et le social, c'est de faire en sorte que cette formidable mutation économique, technologique et de services dont j’ai parlé entraîne aussi une évolution sociale qui est restée en arrière par rapport aux autres, en grande partie d'ailleurs pour des raisons réglementaires puisque cette insuffisante préparation à la concurrence que j'ai mentionnée s'accompagne d'une adaptation insuffisante à la vie ordinaire et quotidienne des entreprises. Songez que la plupart de mes accords, lorsque j'en passe, doivent être transformés en décret. Ce n'est pas cela, le chemin de fer du XXI siècle, ça ne peut pas être cela, ça ne peut être un corps social vivant si on continue à être contraint par ces aspects juridiques. Moi, je voudrais qu'on soit 
dans le management et dans une relation entre l'interne et l'extérieur de l'entreprise via la relation de clientèle entre l'agent et le client, et que cela conduise à une réconciliation avec toutes les dimensions de la société.

De ces dimensions, plusieurs sont bien assumées par la SNCF, notamment les approches territoriales, technologiques, écologiques. En revanche nous manque la compréhension réciproque de l'ensemble des enjeux de société. C'est pour moi une grande chance d'être le président de l'entreprise au moment où elle est confrontée à ces trois révolutions nécessaires. Tous les patrons qui m'entourent, que j'ai mis en place, avec qui $j$ 'ai la chance de travailler, ressentent avec moi la gravité de ce moment historique. Votre association doit sans doute relativiser ce caractère ; pour moi, $j$ 'ai la faiblesse de considérer que trois aussi fortes révolutions en même temps ont un caractère plus historique que d'autres et que ce moment est extraordinairement motivant.

Je terminerai en disant que la dimension de ces évolutions n'est plus celle de la France. Dans vingt ans, ce ne sera plus l'Association pour l'histoire des chemins de fer en France qui nous recevra puisque cette dimension n'aura plus autant de sens. La dimension du chemin de fer est européenne, les trafics sur lesquels j'ai intérêt à faire circuler mes trains de marchandises viennent d'Anvers beaucoup plus que du Havre. Environ $25 \%$ de mon chiffre d'affaires pour la grande vitesse est fait à l'international. Le potentiel de développement le plus important se trouve dans la dimension internationale. Mon ambition est européenne - elle est mondiale pour certains aspects seulement du transport de marchandises par l'intermédiaire de Géodis dont nous sommes l'actionnaire industriel - quand il s'agit d'alimenter les flux transportés par le chemin de fer.

D'ici quelques années, l'histoire du chemin de fer dépassera peut-être le chemin de fer français ou européen, pour être celle de la mobilité écologique et du rôle du ferroviaire dans cette mobilité écologique. Très peu de mes clients me disent : «Je veux acheter du train. » Mes clients me disent : «Je veux acheter de la mobilité. » Ils choisissent le train, quand le train est compétitif ou quand ils y sont contraints. Tout ce que nous pourrons faire pour amener les clients vers le train est ce que nous pourrons faire de mieux pour le ferroviaire : cela va nous mener dans des lieux inhabituels. Par exemple, l'autorisation que j'ai donnée à Géodis d'acheter une commission de transport sur le port de Shanghaï fera partie de l'histoire du chemin de fer français. Autre exemple, on m'a suggéré d'acheter l'an prochain une société de distribution de billets de tourisme en Europe pour en quelque sorte rendre européen www.voyagessncf.com. C'est ainsi que je veux développer le chemin de fer, en investissant 
dans la distribution. De même, $j$ 'ai refusé de vendre les parkings, qui resteront donc dans le patrimoine de la SNCF, car je pense que c'est un levier essentiel du développement du trafic TER.

Voilà donc comment, quand nous nous projetons dans l'avenir, notre chemin de fer prend d'autres dimensions, une autre dimension géographique, d'autres dimensions économiques et, finalement, de services. Je ne sais pas si mes propos rendent compte de ce que vous, les spécialistes, les historiens, pouvez déjà percevoir par votre analyse intellectuelle des faits, mais, en tout cas, c'est ce que nous vivons, nous, aujourd'hui, à la tête de ce morceau-là de l'histoire du chemin de fer français. 